\title{
Esclerosis hepatoportal como causa de hipertensión portal no cirrótica en un paciente colombiano infectado con VIH
}

\section{Hepatoportal Sclerosis as a Cause of Portal Hypertension in a Colombian HIV patient without Cirrhosis}

\author{
Luis Eduardo Barrera Herrera, ${ }^{1}$ Mónica Tapias M., ${ }^{2}$ Víctor Idrovo V., ${ }^{2}$ Enrique Andrade R., ${ }^{3}$ Rocío del Pilar López R. ${ }^{4}$
}

\footnotetext{
1 Departamento de Patología y Laboratorios, Hospital Universitario Fundación Santa Fe de Bogotá. Bogotá, Colombia

2 Servicios de Gastroenterología, Hepatología y Trasplantes, Hospital Universitario Fundación Fe de Bogotá. Bogotá, Colombia

3 Departamento de Patología y Laboratorios, Hospital Universitario Fundación Santa Fe de Bogotá, Facultad de Medicina Universidad de los Andes y Universidad Nacional de Colombia. Bogotá, Colombia

${ }^{4}$ Departamento de Patología y Laboratorios, Hospital Universitario Fundación Santa Fe de Bogotá y Facultad de Medicina de la Universidad de los Andes. Bogotá, Colombia

Presentación previa: póster (reporte de caso): Esclerosis hepatoportal como causa de hipertensión portal no cirrótica, en paciente infectado por el virus de la inmunodeficiencia humana (HIV). López R., Tapias M., Andrade R. XXXIX Congreso Colombiano de Patología, SOCOPAT, Sociedad Colombiana de Patología, Santa Marta, Colombia, 2012.
}

Fecha recibido: $26-06-14$

Fecha aceptado: 02-02-15

\begin{abstract}
Resumen
Antecedentes: la esclerosis hepatoportal se manifiesta como hipertensión portal no cirrótica. Su etiología parece estar relacionada con alteraciones idiopáticas en la microvasculatura hepática. Las manifestaciones de la esclerosis hepatoportal incluyen sangrado de vías digestivas altas, pancitopenia, esplenomegalia e hipertensión portal no cirrótica. Presentamos el primer caso reportado en Colombia de esclerosis hepatoportal en un paciente con serología positiva para el virus de la inmunodeficiencia humana (VIH). Métodos: paciente masculino de 60 años de edad, VIH-positivo, quien ingresa a nuestra institución por hemorragia de vías digestivas alta (várices esofágicas y fúndicas) y ascitis, cuyo manejo requirió la toma de biopsia hepática. Resultados: se realizó biopsia Trucut de hígado que evidenció la presencia de 6 a 8 espacios porta con parénquima arquitectónico conservado que demostró fibrosis perivenular y dilatación sinusoidal pericentral severa. Conclusión: la esclerosis hepatoportal es una causa de morbilidad en pacientes VIH-positivos. Debe considerarse en cada paciente que manifiesta hipertensión portal no cirrótica asociada con hemorragia de la vía digestiva alta. Sin embargo, una investigación adicional es imprescindible con el fin de describir la relación entre el desarrollo de alteraciones intrahepáticas (microtrombosis), la patogénesis del VIH y el uso de terapia antirretroviral, particularmente el uso de didanosina.
\end{abstract}

\section{Palabras clave}

Hígado, biopsia Trucut, esclerosis hepatoportal, hipertensión portal no cirrótica, virus de la inmunodeficiencia humana, terapia antirretroviral.

\begin{abstract}
Background: Hepatoportal sclerosis manifests as non-cirrhotic portal hypertension. Its etiology appears to be related to alterations in the idiopathic micro-vasculature of the liver. Manifestations of hepatoportal sclerosis include upper gastrointestinal bleeding, pancytopenia, splenomegaly and non-cirrhotic portal hypertension. We present the first reported case of hepatoportal sclerosis in Colombia which occurred in an HIV positive patient. Methods: A 60-year-old male HIV patient positive was admitted to our institution because of ascites and upper digestive tract bleeding due to esophageal and fundal varices. Management required taking a liver biopsy. Results: A Tru-Cut biopsy needle was used to take a liver biopsy sample percutaneously. The biopsy revealed six to eight portal tracts with preserved architectural parenchyma, perivenular fibrosis and severe pericentral sinusoidal dilatation. Conclusions: Hepatoportal sclerosis is a cause of morbidity in HIV-positive patients and should be considered in each patient manifesting non-cirrhotic portal hypertension associated with upper gastrointestinal bleeding. However, further research is necessary to describe the relationship between the development of intrahepatic alterations (microthrombosis), HIV, and the use of anti-retroviral therapy, particularly the use of didanosine.
\end{abstract}

\section{Keywords}

Tru-cut liver biopsy, hepatoportal sclerosis, non-cirrhotic portal hypertension, human immunodeficiency virus, anti-retroviral therapy. 


\section{INTRODUCCIÓN}

Las principales causas de morbilidad y mortalidad en pacientes con enfermedad hepática y serología positiva para virus de la inmunodeficiencia humana (VIH) son secundarias a la infección por el virus de la hepatitis B y/o C, siendo también frecuente la esteatohepatitis alcohólica y no alcohólica, la toxicidad por terapia antirretroviral (TARV) y la hipertensión portal no cirrótica (HPNC). Aunque con menor frecuencia, la HPNC ha sido descrita en pacientes con serología positiva para VIH quienes además tienen en común la TARV prolongada con didanosina (1). En este sentido y con al menos 100 casos reportados en la literatura, la hiperplasia nodular regenerativa y la esclerosis hepatoportal (EHP) son los hallazgos más comunes (2). Aunque el patrón de daño hepático relacionado con la TARV no está bien establecido, se han descrito casos de pacientes con HPS que requirieron trasplante hepático $(3,4)$.

\section{PACIENTE Y MÉTODO}

Paciente de 60 años de edad, sexo masculino y serología positiva para VIH de más de 15 años de evolución, diagnosticado luego de un episodio de histoplasmosis diseminada. Ingresa al servicio de urgencias por episodio de hematemesis secundario a sangrado de várices esofágicas y hemorragia fúndica asociadas con la presencia de ascitis. Los estudios paraclínicos de ingreso mostraron: transaminasa (TGO-AST), $58 \mathrm{U} / \mathrm{L}$ (5-34 U/L); transaminasa (TGP-ALT), $50 \mathrm{U} / \mathrm{mL}$ (<55 U/L); fosfatasa alcalina, 164 $\mathrm{U} / \mathrm{L}$ (40-50); bilirrubina total, $46 \mathrm{mg} / \mathrm{dL}(0,2-1 \mathrm{mg} / \mathrm{dL})$; directa, $0,17 \mathrm{mg} / \mathrm{dL}(<0,3 \mathrm{mg} / \mathrm{dL})$; indirecta, $0,29 \mathrm{mg} /$ $\mathrm{dL}$; plaquetas, $168(10)^{3} / \mathrm{mm}^{3}\left(150-450(10)^{3} / \mathrm{mm}^{3}\right)$; INR, 1,05; carga viral de VIH, hepatitis B y hepatitis C negativa; conteo de linfocitos CD4 280 células. La medicación TARV incluía zidovudina, didanosina y efavirenz, la cual recibió durante más de 14 años. Los medicamentos adicionales para la hipertensión arterial, gastritis, hipercolesterolemia y espondilitis incluían: metoprolol, aspirina, sulfasalazina, atorvastatina, clopidogrel, alopurinol y colchicina desde hace 3 años.

Se realizó endoscopia de vías digestivas que reveló la presencia de várices esofágicas, las cuales fueron ligadas. Se inició heparina de bajo peso molecular (enoxaparina) $40 \mathrm{mg} /$ día. La ecografía Doppler (figura 1A y 1B) de hígado evidenció hígado con signos de hepatopatía crónica, sin lesiones focales y sin cirrosis; trombosis de la vena porta que se extendía desde el confluente venoso esplenomesentérico hasta las ramas intraparenquimatosas portales; adecuada permeabilidad de las venas hepáticas; signos de hipertensión portal dados por la discreta cantidad de líquido perihepático; esplenomegalia y engrosamiento de las paredes vasculares. Se realizó el diagnóstico presuntivo de HPNC.

El estudio de líquido ascítico reveló gradiente de albúmina mayor de $1,1 \mathrm{~g} / \mathrm{dL}$, negativo para infección. Los cultivos fueron negativos para gérmenes comunes, incluidas las micobacterias y los hongos. Se suspendieron los posibles medicamentos causantes de la toxicidad hepática, que incluían atorvastatina y sulfasalazina. Se realizó biopsia hepática sin complicaciones (figura 2). El paciente evolucionó con episodios de encefalopatía de muy probable origen hepático secundario a la HPNC, siendo sus manifestaciones clínicas bradipsiquia, alteración del ciclo sueño-

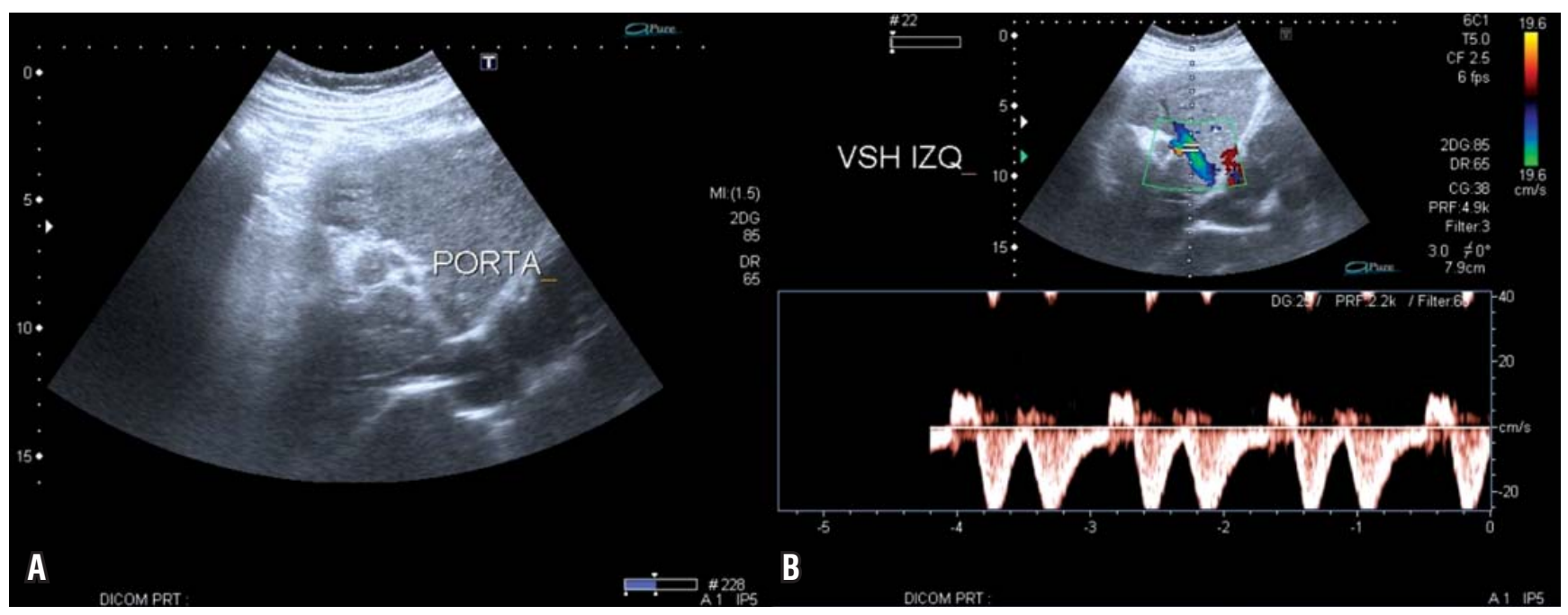

Figura 1. Ecografía Doppler de hígado. A. Trombosis de la vena porta. B. Compromiso del aspecto más superior de la vena mesentérica superior, la vena porta principal y la ramas portales intrahepáticas. 
vigilia y de la memoria. Durante su hospitalización, el paciente evolucionó con mejoría de los síntomas. Después de 1 año presentó hemorragia digestiva masiva y falleció.

\section{RESULTADOS}

A partir del tejido obtenido por biopsia Trucut, se realizaron cortes seriados coloreados con $\mathrm{H} \& \mathrm{E}$ y estudios de histoquímica para hierro, PAS-diastasa, tricrómico de Masson, aldehído fucsina, Kinyoun y reticulina.

\section{Estudio microscópico}

El parénquima hepático mostró preservación de su arquitectura. Se visualizaron 8 espacios porta con fibrosis perivenular portal marcada, acompañada de ocasionales células linfoides, sin necrosis de hepatocitos ni evidencia de colestasis aguda o crónica. El hallazgo más relevante fue la dilatación sinusoidal pericentral (zona 3) (figuras 2A y 2B). Las células de Kupffer hipertróficas con pigmento marrón e intensamente reactivas con PAS-diastasa. En la zona 3 se identificó un pigmento citoplasmático de lipofuscina, positivo para la coloración de Kinyoun, débilmente positivos con PAS-diastasa y aldehído fucsina, hierro y cobre negativos. El tricrómico de Masson muestra fibrosis perivenular a nivel portal (figuras $2 \mathrm{C} \mathrm{y} 2 \mathrm{D}$ ) con atrofia focal trabecular debida a hiperplasia nodular regenerativa.

\section{DISCUSIÓN}

La HPNC es una rara condición con manifestaciones clínicas de hipertensión portal que incluyen várices esofágicas, gastropatía hipertensiva, ascitis, esplenomegalia, entre otros, todos ellos en ausencia de cirrosis. La causa de la derivación portosistémica no está claramente establecida y puede presentarse encefalopatía de origen hepático en ausencia de cirrosis; esta, a su vez, puede manifestarse principalmente por síntomas neuropsiquiátricos. Por otro lado, la trombosis de la porta puede en ocasiones ser la causa de hipertensión portal, lo que lleva a episodios de encefalopa-

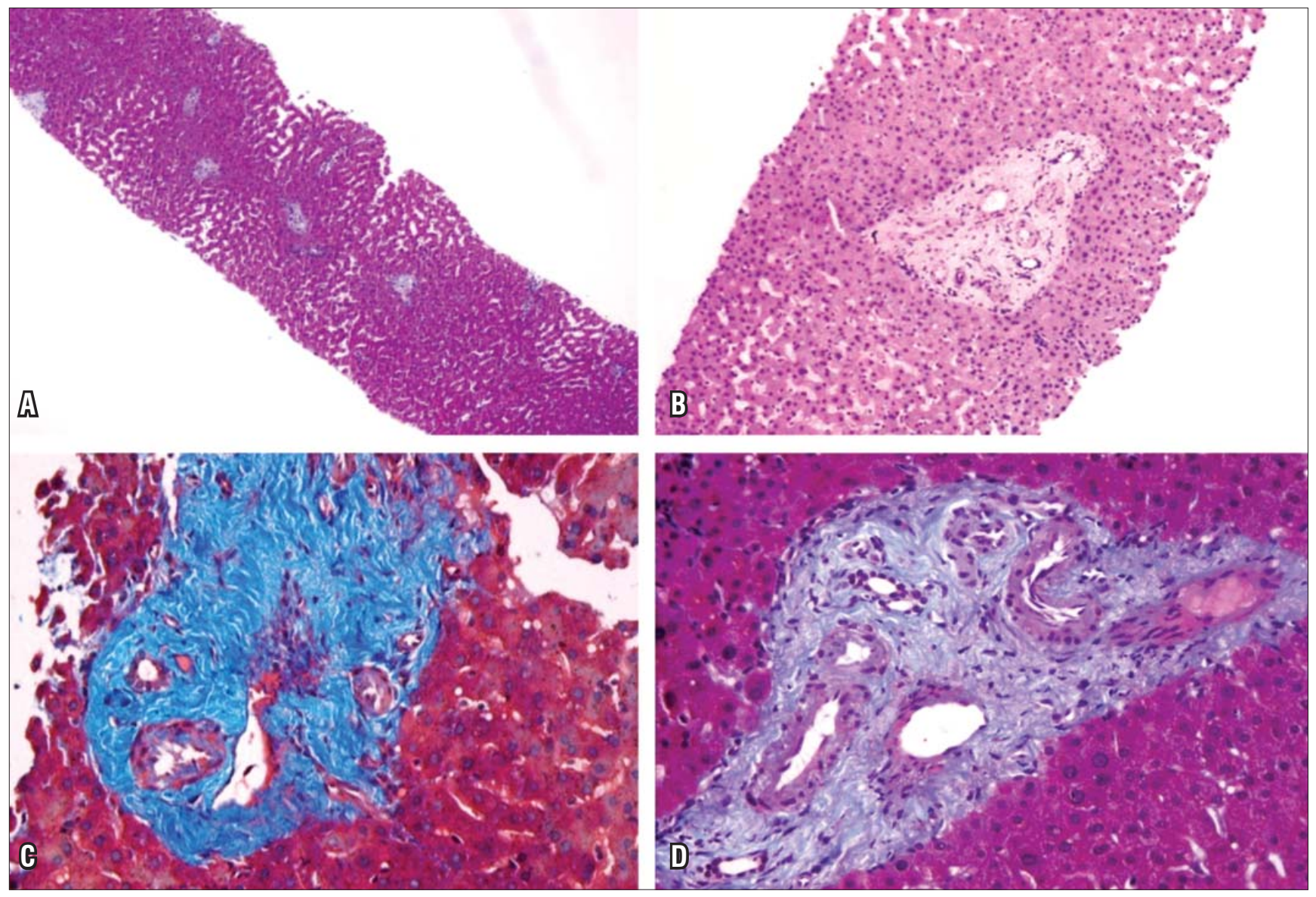

Figura 2. Biopsia hepática. A. HE $x$ 10: parénquima con preservación arquitectural y marcada dilatación sinusoidal pericentral. B. HE $x 40$ : espacio porta con importante esclerosis perivenular portal sin evidencia de inflamación; hay dilatación sinusoidal en zona 3. C y D. Tricrómico de Masson $x$ 40: resalta la fibrosis perivenular. 
tía de intensidad variable. La hipótesis más aceptada de su mecanismo es el edema de las células astrocitarias, producido por el metabolismo del amonio en el cerebro (5-8).

En cuanto a las entidades que causan HPNC, se ha descrito la trombosis de la vena porta, la hiperplasia nodular regenerativa, la cirrosis septal incompleta, los distintos tipos de vasculitis y la esquistosomiasis (9-12). Aunque la etiología de la HPNC no ha sido establecida, se proponen mecanismos multifactoriales para explicar su patogénesis. Se han descrito alteraciones en la vasculatura portal prehepática por lesión celular endotelial por VIH y por el uso de medicamentos como la didanosina (13), así como estados protrombóticos debidos a la infección por VIH como causa directa de anticuerpos de proteína $S$, que llevan a la deficiencia de proteína $S$ y que resultan en obliteración de las vénulas portales y regeneración e hiperplasia hepática (14). Igualmente la hipertensión portal presinusoidal aparece como perfil hemodinámico de la HPNC, que muestra un gradiente de presión venosa hepática normal o ligeramente elevada $(<10 \mathrm{~mm} \mathrm{Hg})(15)$.

Durante los últimos 10 años encontramos varios reportes de pacientes VIH-positivos con síntomas de HPNC, algunos de los cuales presentaron evolución tan severa que requirieron trasplante hepático. Se ha expuesto cómo la relación entre el VIH y la HPNC parece ser multifactorial. Los estados protrombóticos y el tratamiento con TARV, especialmente didanosina, se han postulado como la causa de esta enfermedad en pacientes VIH-positivos, siendo además la didanosina la asociada con mayor frecuencia en la literatura $(1,4)$, aparentemente a través de la dosificación acumulativa o de los mecanismos idiosincrásicos (15); incluso, los polimorfismos en genes implicados en el metabolismo de la didanosina podrían predisponer a enfermedad hepática venooclusiva (16).

Poco se sabe sobre el mecanismo exacto de daño de los hepatocitos por TARV. Los casos publicados muestran cómo, hasta el momento, la cirrosis idiopática con hipertensión portal comienza con una lesión en los vasos más pequeños y progresa hasta producir cambios en la arquitectura del parénquima hepático con hiperplasia nodular regenerativa (1). Mientras que en la HPNC la trombosis portal es un hallazgo común (17), que puede ser causa y consecuencia de la descompensación hepática (16), la EHP se ha descrito como el mecanismo subyacente a la HPNC en pacientes VIH-positivos, el cual se atribuye a microtrombosis intrahepática o a las anormalidades de la fibrogénesis hepática por ART o como parte de los daños por VIH (2). En nuestro caso, los hallazgos histológicos descritos de fibrosis portal y dilatación sinusoidal fueron las únicas alteraciones encontradas en el estudio microscópico que explican la obstrucción del flujo venoso y, por ende, los síntomas asociados.
Mundialmente la EHP ha demostrado ser una clara causa de morbilidad en pacientes VIH-positivos y dado su alto potencial de mortalidad, se tiene que tener en cuenta en todo paciente que manifiesta HPNC con hemorragia digestiva alta (18). En la literatura, la mayoría de los casos reportados incluye pacientes que presentan hemorragia digestiva alta cuyas pruebas hepáticas son normales y que además tienen en común manejo TARV con didanosina durante períodos prolongados (19), este último relacionado con daño mitocondrial y consiguiente daño a tejido vascular endotelial hepático (17).

\section{CONCLUSIÓN}

A pesar de que hace algunos años la enfermedad hepática de causa desconocida en pacientes seropositivos para VIH era poco común, en la actualidad es cada vez más informada, siendo la HPNC el hallazgo más frecuente. Presentamos el primer caso de EHP en un paciente con VIH reportado en Colombia, destacando además la importancia en la interpretación microscópica con la correlación clínica y los estudios radiológicos, que en nuestro caso en conjunto explicaron los signos y síntomas de la HPNC y en el paciente correspondieron a una EHP. Se debe tener presente incluir en el diagnóstico diferencial de pacientes con síntomas neuropsiquiátricos e HPNC la posibilidad de una encefalopatía portosistémica.

Por último, el presente reporte destaca la necesidad de desarrollar futuras investigaciones con el fin de establecer la relación entre la patogénesis del VIH, el tratamiento con TAR y las alteraciones hepáticas, especialmente sobre la escasa evidencia para recomendar terapia de anticoagulación y evaluación suplementaria de estados protrombóticos en estos pacientes.

\section{Conflicto de intereses}

Los autores declaran que no existe ningún conflicto de interés con respecto a la publicación de este artículo.

\section{REFERENCIAS}

1. Kovari H, Ledergerber B, Peter U, Flepp M, Jost J, Schmid $\mathrm{P}$, et al. Association of noncirrhotic portal hypertension in HIV-infected persons and antiretroviral therapy with didanosine: a nested case-control study. Clin Infect Dis Off Publ Infect Dis Soc Am 2009;49(4):626-35.

2. Schiano TD, Kotler DP, Ferran E, Fiel MI. Hepatoportal sclerosis as a cause of noncirrhotic portal hypertension in patients with HIV. Am J Gastroenterol 2007;102(11):2536-40.

3. Isabel Fiel M, Thung SN, Hytiroglou P, Emre S, Schiano TD. Liver failure and need for liver transplantation in patients 
with advanced hepatoportal sclerosis. Am J Surg Pathol 2007;31(4):607-14.

4. Krasinskas AM, Eghtesad B, Kamath PS, Demetris AJ, Abraham SC. Liver transplantation for severe intrahepatic noncirrhotic portal hypertension. Liver Transplant Off Publ Am Assoc Study Liver Dis Int Liver Transplant Soc 2005;11(6):627-34; discussion 610-1.

5. Fukushima K, Kurozumi M, Kadoya M, Ikeda S. Portalsystemic encephalopathy in a non-cirrhotic patient. BMJ Case Rep 2009;2009:bcr2007121822.

6. Fukushima K, Kurozumi M, Kadoya M, Ikeda S. Neurological picture. Portal-systemic encephalopathy in a non-cirrhotic patient. J Neurol Neurosurg Psychiatry 2008;79(1):96.

7. Watanabe A. Portal-systemic encephalopathy in non-cirrhotic patients: classification of clinical types, diagnosis and treatment. J Gastroenterol Hepatol 2000;15(9):969-79.

8. Mínguez B, García-Pagán JC, Bosch J, Turnes J, Alonso J, Rovira A, et al. Noncirrhotic portal vein thrombosis exhibits neuropsychological and MR changes consistent with minimal hepatic encephalopathy. Hepatol Baltim Md 2006;43(4):707-14.

9. Reshamwala PA, Kleiner DE, Heller T. Nodular regenerative hyperplasia: not all nodules are created equal. Hepatol Baltim Md 2006;44(1):7-14.

10. Sciot R, Staessen D, Van Damme B, Van Steenbergen W, Fevery J, De Groote J, et al. Incomplete septal cirrhosis: histopathological aspects. Histopathology 1988;13(6):593603.

11. Nayak NC, Ramalingaswami V. Obliterative portal venopathy of the liver. Associated with so-called idiopathic portal hypertension or tropical splenomegaly. Arch Pathol 1969;87(4):359-69.
12. Ludwig J, Hashimoto E, Obata H, Baldus WP. Idiopathic portal hypertension; a histopathological study of 26 Japanese cases. Histopathology. 1993;22(3):227-34.

13. Saifee S, Joelson D, Braude J, Shrestha R, Johnson M, Sellers $\mathrm{M}$, et al. Noncirrhotic portal hypertension in patients with human immunodeficiency virus-1 infection. Clin Gastroenterol Hepatol Off Clin Pract J Am Gastroenterol Assoc 2008;6(10):1167-9.

14. Alvarez Díaz H, Mariño Callejo A, García Rodríguez JF. Non-cirrhotic portal hypertension in human immunodeficiency virus-infected patients: a new challenge in antiretroviral therapy era. Open AIDS J 2011;5:59-61.

15. Chang P-EJ, Garcia-Pagan J-C. Idiopathic noncirrhotic portal hypertension in HIV-infected patients. Clin Infect Dis Off Publ Infect Dis Soc Am 2010;50(1):127-8; author reply 128-9.

16. Mendizabal M, Craviotto S, Chen T, Silva MO, Reddy KR. Noncirrhotic portal hypertension: another cause of liver disease in HIV patients. Ann Hepatol 2009;8(4):390-5.

17. Walker UA, Setzer B, Venhoff N. Increased long-term mitochondrial toxicity in combinations of nucleoside analogue reverse-transcriptase inhibitors. AIDS Lond Engl 2002;16(16):2165-73.

18. Castellares C, Barreiro P, Martín-Carbonero L, Labarga P, Vispo ME, Casado R, et al. Liver cirrhosis in HIV-infected patients: prevalence, aetiology and clinical outcome. J Viral Hepat 2008;15(3):165-72.

19. Vispo E, Moreno A, Maida I, Barreiro P, Cuevas A, Albertos $S$, et al. Noncirrhotic portal hypertension in HIV-infected patients: unique clinical and pathological findings. AIDS Lond Engl 2010;24(8):1171-6. 